\title{
THE MOTION OF IGE SHEETS AND GLACIERS
}

\author{
By J. F. Nye \\ (H. H. Wills Physics Laboratory, University of Bristol)
}

\begin{abstract}
The distribution of velocity along a vertical line in Greenland is calculated. Almost all the relative shear motion is concentrated at and very near to the bottom, and this result is thought to apply generally in Greenland and Antarctica. It is due to the relatively high temperature of the lowest layers and the strong dependence of creep rate on temperature. The outward velocity being thus almost constant with depth, it is assumed proportional to the $m$ th power of the shear stress on the bed; the results previously obtained by the approximation of perfect plasticity then correspond to $m \rightarrow \infty$. Steady-state solutions for finite $m$ are derived, and non-steady states are discussed by use of Weertman's perturbation method. The steady-state height of an ice sheet is found to be very insensitive to the rate of accumulation. Surface waves in Antarctica are attributed to mountains buried beneath the ice, and it is shown how the height and form of a buried mountain ridge may be calculated from the associated surface disturbance.

The application of a law of sliding to glaciers leads to an expression for the longitudinal strain-rate, hitherto an undetermined parameter.
\end{abstract}

RÉsumé. La distribution de la vitesse le long d'une ligne verticale au Groenland a été calculée. La presque totalité du mouvement relatif de cisaillement est concentrée vers le fond et la partie avoisinante, et on estime que cet état de choses est général au Groenland et dans l'Antarctique. Il est attribuable à la température relativement élevée des couches les plus profondes et au rapport étroit qui existe entre la vitesse de fluage et la température. Comme la vitesse vers l'extérieur est donc à peu près constante en raison de la profondeur, on la suppose proportionnelle à la $m$ ième puissance de la tension de cisaillement sur le lit; les résultats qui avaient été obtenus antérieurement par l'approximation de la plasticité parfaite correspondent alors à $m \rightarrow \infty$. On en déduit des solutions pour l'écoulement régulier (pour l'état stationnaire) et des valeurs finies de $m$ et on examine l'écoulement irrégulier et non-stationnaire au moyen de la méthode de perturbation de Weertman. On constate que la hauteur de l'inlandsis en état d'écoulement stationnaire est fort peu sensible à la vitesse d'accumulation. Les ondulations de surface dans l'Antarctique sont attribuées à la présence de montagnes ensevelies sous la glace et on démontre comment on peut calculer la hauteur et la forme d'une chaîne de montagnes ensevelie d'après les perturbations de la surface.

L'application aux glaciers de la loi de glissement conduit à une expression pour la vitesse de déformation longitudinale qui, jusqu'ici constituait un paramètre indéfini.

Zusammenfassung. Es wird die Geschwindigkeitsverteilung entlang einer Vertikallinie in Grönland berechnet. Fast die ganze Scherbewegung ist am Grund oder sehr nahe am Grund konzentriert, und es wird angenommen, dass dieses Resultat allgemein für Grönland und die Antarktis zutrifft. Es beruht auf der relativ hohen Temperatur der tiefsten Schichten und der von der Temperatur stark abhängigen Kriechgeschwindigkeit. Da somit die nach aussen gerichtete Geschwindigkeit per Tiefe fast konstant ist, wird sie als die $m$ te Potenz der Scherspannung am Bett bemessen; die Resultate, die vorher durch Annäherung von perfekter Plastizität erhalten wurden, entsprechen dann $m \rightarrow \infty$. Ergebnisse im stationären Fliessen werden für begrenztes $m$ abgeleitet, und nicht-stationäre zustände werden mit Hilfe der Weertmanschen Störungsmethode besprochen. Es wurde gefunden, dass die Höhe einer Eisfläche im stationären Zustand gegen die Anhäufungsgeschwindigkeit sehr unempfindlich ist. Oberflächenwellen in der Antarktis werden unter dem Eis begrabenen Bergen zugeschrieben, und es wird gezeigt, wie die Höhe und Form einer begrabenen Bergkette von der damit verbundenen Oberflächenstörung berechnet werden kann.

Die Anwendung eines Gleitgesetzes auf Gletscher führt zu einem Ausdruck für die Längsdehnungsgeschwindigkeit, ein bisher unbestimmter Parameter.

\section{InTRODUCTION}

The velocity of a point on the surface of an ice sheet is made up, in general, of two contributions: one arises from the sliding of the ice sheet over its bed, and the other from differential shear motion within the bulk of the ice. In Section 2 of this paper we argue that in the ice sheets of Antarctica and Greenland the motion may be regarded for most purposes as made up entirely of sliding. This is a considerable simplification, and the remainder of the ice sheet theory which follows in Sections 3.1 to 3.5 is based upon it. In these sections, by using a suitable law of sliding, we study the flow of a general ice sheet of irregular outline on an irregular bed in both a steady and a non-steady state. The theory is set up in such a way that previous results, obtained from the approximation of perfect plasticity, appear as special
cases.

In other cases, however, including perhaps most glaciers, we cannot ignore the differential shear motion. The application of the law of sliding to these cases (Section 4) makes it possible to calculate the longitudinal strain-rate, which has hitherto been an undetermined parameter of the theory. 
Readers who prefer to omit Section 2 will find that the rest of the paper may be read independently.

\section{Galgulation for Greenland and Reasons for Using a Law of Sliding}

In the approximation of perfect plasticity the difference of velocity between the top and bottom of a large ice sheet is only a small fraction of the total velocity (Ref. I, p. 57I). With the more realistic theory of reference 2 it is possible to check this result.

The relevant equations (Ref. 2, p. 129) are as follows:

$$
\dot{\gamma}=-2 \sqrt{ }\left(\dot{\epsilon}^{2}-r^{2}\right), \quad \rho g y \sin \alpha=\frac{\tau}{\dot{\epsilon}} \sqrt{ }\left(\dot{\epsilon}^{2}-r^{2}\right),
$$

where $\dot{\gamma}$ is the rate of shear strain (rate of change of forward velocity with respect to depth) at depth $y$ below the surface, $r$ is the modulus of the longitudinal strain-rate (uniform with depth), $\rho$ is the mean density of the ice between the surface and the depth $y$, and $\alpha$ is the slope of the upper surface. $\tau$ is the "effective shear stress" and $\dot{\epsilon}$ is the "effective strain-rate", two parameters which are connected by the flow law

$$
\dot{\epsilon}=f(\tau)
$$

the flow law depends on the temperature. The temperature at each depth, and the flow law, are supposed known. $r$ is regarded as a given parameter. The calculation consists of choosing a value of $y$, putting in the flow law appropriate to this depth, and solving equations (I) numerically for $\dot{\gamma}$. Thus $\dot{\gamma}$ is found as a function of $y \cdot \int_{0}^{y} \dot{\gamma} d y$ then gives the required distribution of velocity with $y$.

We have carried out the calculation for a particular point in north Greenland: station $\operatorname{A~}_{125}\left(78^{\circ}\right.$ N., $53^{\circ}$ W.) on the traverse of the British North Greenland Expedition, 3 for which $\alpha=9 . I^{\prime}$ and the total depth $h$ is $2,300 \mathrm{~m}$. $\rho$ is taken as $0.90 \mathrm{gm} . / \mathrm{cm}$. ${ }^{3}$. Since $r \sim a / h$, where $a$ is the rate of accumulation* we have estimated $r$ as $0 \cdot 12 / 2,300=5 \times 10^{-5} \mathrm{yr}^{-1}$. For the variation of temperature with depth we have used the curve shown in Fig. I. This is not precisely calculated, but has been drawn to be consistent with Robin's calculations. ${ }^{4}$ The temperature curve enables us to find from Glen's experiments 5 the flow law applicable to each depth. Generalizing Glen's results to three-dimensional states of stress, we put $\dot{\epsilon} \propto \tau^{n}$ for a given temperature. For $\dot{\epsilon}$ we have used the quasi-viscous creep rate $(n=4 \cdot 2)$, and have assumed that the temperature dependence of the quasi-viscous creep rate is the same as that found experimentally for the minimum creep rate. We should say at once that this use of Glen's results involves extrapolating the experimental data by a factor of $\mathrm{IO}^{-2}$ to $\mathrm{IO}^{-3}$ in strain-rate. The experimental temperature range was $0^{\circ} \mathrm{C}$. to $-13^{\circ} \mathrm{C}$. and the range used in the calculation is $-4^{\circ} \mathrm{C}$. to $-23^{\circ} \mathrm{C}$.

We have made no allowance for any effect of hydrostatic pressure on the flow law. Rigsby finds ${ }^{6}$ that there is no effect provided the temperature is taken relative to the pressure melting point rather than to $0^{\circ} \mathrm{C}$. The pressure melting point at a depth of $2,300 \mathrm{~m}$. is $-1 \cdot 5^{\circ} \mathrm{C}$. Therefore if this correction were applied our result would correspond to a corrected temperature distribution $\theta-\mathrm{I} \cdot 5(y / h)$ degrees Centigrade, where $\theta$ is the temperature shown in Fig. I.

Equations (I) cannot be solved directly, with $\dot{\epsilon}$ as a parameter, because the flow law depends on temperature, and therefore on $y$. To obtain $\dot{\gamma}(y)$ we therefore had to use a method of successive approximation. $\dagger$ The result for $\dot{\gamma}(y)$ is shown in Fig. 2, and the result for $\tau(y)$ in Fig. 3. It is then a simple matter to calculate the stress components $\sigma_{x}, \sigma_{y}, \tau_{x y}$ from equations

* In the absence of direct measurement of $r$ a more exact value could be obtained from equation (33) of Section 4 .

$\dagger$ The sequence is as follows. Start with a value of $y$; read off the temperature; now guess $\dot{\epsilon}$; hence $\tau$; hence $\rho g y \sin \alpha$; hence $y$. This last value of $y$ is different from the one we began with. So we now make a better guess at $\dot{\epsilon}$ and repeat the sequence until it is self-consistent. A maximum of five repeats was sufficient in all cases. Thus we find $\dot{\epsilon}(y)$ and hence $\dot{\gamma}(y)$. 
Temperature $\left({ }^{\circ} \mathrm{C}\right)$

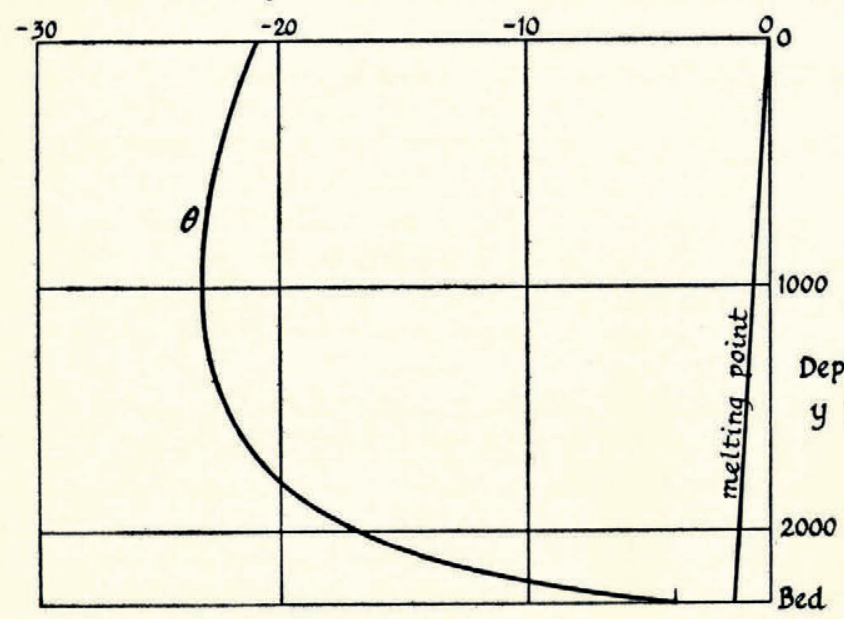

Fig. I. Temperature-depth relation at station A I25 in Greenland assumed for the calculation of velocity

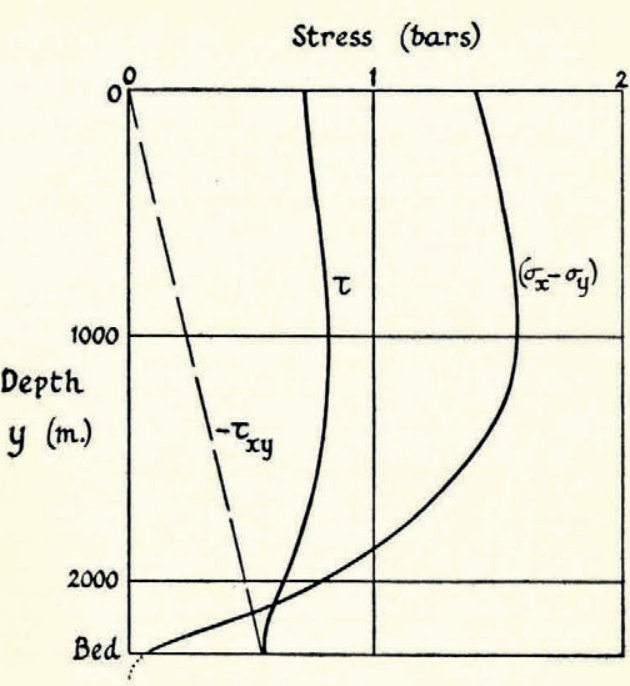

Fig. 3. The effective shear stress $\tau$, the shear stress $\tau_{x y}$, and the stress difference $\sigma_{x}-\sigma_{y}$, all as functions of depth at station A 125 in Greenland. $\sigma_{y}$ (not plotted) varies nearly linearly from o at the surface to -203 bars at the bed

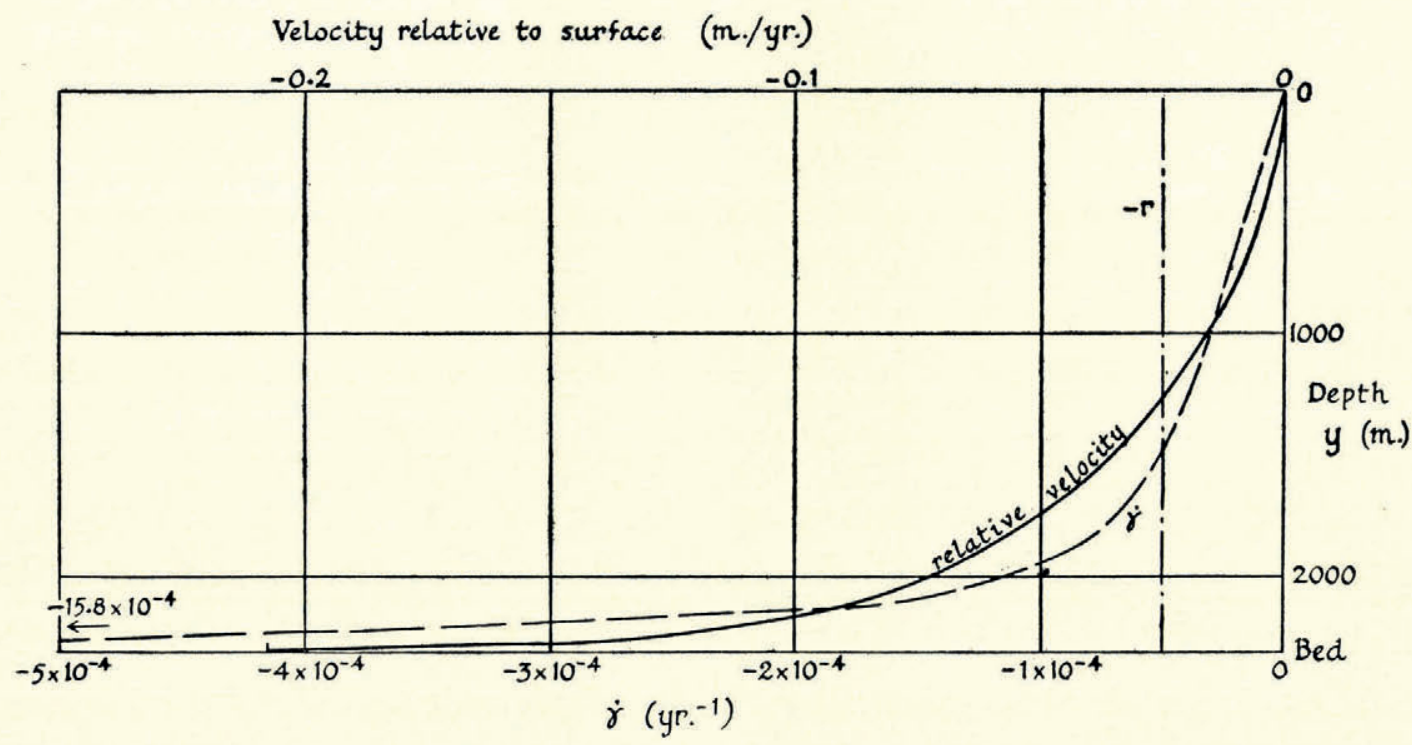

Fig. 2. The rate of longitudinal strain $r$, the rate of shear strain $\dot{\gamma}$ and the differential velocity, all as functions of depth at station $A$ I25 in Greenland

(I9) of reference 2, and $\sigma_{x}-\sigma_{y}$ and $\tau_{x y}$ are plotted in Fig. 3. The velocity distribution, obtained by numerical integration of $\dot{\gamma}$, is shown in Fig. 2.

The extrapolation of the flow law to low strain-rates, and the fact that the temperature distribution is to some extent guessed, mean that the precise numerical results of the calculation have little significance. However, there are certain extreme features of the result which it is thought are significant, and which we now discuss under headings $(a)-(d)$ below.

(a) The uniformity of $\tau$. Consider first the distribution of $\tau$, the effective shear stress, with 
depth (Fig. 3). It is 0.700 bars at the surface, its maximum value is 0.813 bars and its minimum is 0.537 bars. This rather small variation means that the approximation of perfect plasticity ( $\tau=$ constant) happens to be a reasonable one for this case. Thus the calculated curves for $\sigma_{x}-\sigma_{y}$ and for the velocity may be compared with quadrants of ellipses. In the approximation of perfect plasticity the total differential motion between top and bottom is of the order of twice the rate of accumulation, that is, $24 \mathrm{~cm}$. $/ \mathrm{yr}$. In the present calculation it is $2 \mathrm{I} \mathrm{cm}$./yr., which is not significantly different. The fact that perfect plasticity provides a reasonably good approximation seems to be a coincidence brought about by the temperature distribution; if, for example, the upper layers happened to be much colder, $\tau$ would be much greater there and $\tau$ constant would cease to be a good approximation.

$\sigma_{x}$ at the surface is $+\mathrm{I} \cdot 4$ bars, treating the firn as ice, and the depth of the tensile layer (using $\rho=0.65$ ) is $22 \mathrm{~m}$. Thus, in spite of the very small rate of extension of the surface, there is a considerable tensile stress because of the low temperature. One would hardly expect crevasses to appear, however, because of the small rate of opening. For example, with $r=5 \times$ IO $^{-5} \mathrm{yr}^{-1}$ a series of cracks $100 \mathrm{~m}$. apart would each only open up about $5 \mathrm{~mm}$. in the course of one year, and they would be swiftly buried.

(b) Relative importance of the longitudinal stretching and the shear motion. Consider now the relative magnitudes of $r$ and $|\dot{\gamma}| .|\dot{\gamma}|$ is of course zero at the surface, and $\left|\frac{1}{2} \dot{\gamma}\right|$ remains less than $r$ down to a depth of I,930 m.; below this, however, it increases very rapidly, and at the lowest depth of $2,300 \mathrm{~m}$. $\left|\frac{1}{2} \dot{\gamma}\right|$ is 16 times greater than $r .|\dot{\gamma}|$ would in any case increase with a high power of the depth, but this effect is much enhanced by the high temperature of the lowest layers. If we had assumed that the temperature at the bottom reached the pressure melting point $\left(-\mathrm{I} \cdot 5^{\circ} \mathrm{C}\right.$.) instead of only $-4^{\circ} \mathrm{C}$., $|\dot{\gamma}|$ at the bottom would have been even greater. Hence, in any event, $|\dot{\gamma}| \gg r$ in the lowest layers. This means that we could have approximately calculated their rate of shearing $\dot{\gamma}$ by ignoring $r$ and assuming laminar flow.

(c) Relative importance of differential velocity within the ice and bed slip. If we assume a steady state, the average outward velocity over the section considered is I I m./yr., which is 50 times the calculated differential motion between top and bottom. Thus on our calculation the outward motion consists almost entirely of sliding on the bottom. This result might well have been different, however, if we had assumed a higher temperature for the lowest layers, for, as we have seen, $\dot{\gamma}$ is extremely sensitive to the temperature in these layers. Thus, whether the motion at the bottom actually consists mainly of sliding or mainly of rapid shear in the lowest layers of ice is problematic. The question in any case is of doubtful significance, because the distinction between the two processes becomes rather artificial when the thickness of the layers involved is comparable, as it probably is, with the height of the natural irregularities of the bed and with the thickness of any bottom moraine carried by the ice.

(d) Concentration of shearing at the bottom. It is clear that whatever shear distortion there is is largely concentrated in a thin bottom layer perhaps roo m., or much less, thick. Thus relative motion, whether between ice and ice, or between ice and rock, is essentially concentrated at the bottom. This is our main conclusion. We can see from the calculation that it is a necessary consequence of the temperature distribution. The bottom layers are the warmest, and the gradient of temperature in them, due to geothermal and frictional heat, is fairly large; the strain-rate is strongly dependent on temperature, and hence the conclusion follows.

Depending, as it does, on the broad features of the temperature distribution, this result presumably applies to all large polar ice sheets. We may therefore think of their outward motion as made up entirely of bottom sliding, if we include in this term the shear in the lowest layer of ice. Allied with the sliding, of course, is the downward and outwards spreading represented by the strain-rate $r$.

(e) Assumptions made in subsequent theory. The theory which follows is based upon the approximation that the outward velocity of the ice $u$ is uniform with depth and is determined 
by the shear stress $T$ at the bed. In particular, we assume that, at a given place on the bed

$$
u=\left(\frac{T}{A}\right)^{m} \quad(T>0),
$$

where $A$ and $m$ are constants determined in general by the roughness of the bed, by the temperature, and by the constitution of the ice. This is the form calculated by Weertman 7 for the sliding velocity when the ice in contact with the bed is at the pressure melting point, in which case $m=(n+\mathrm{I}) / 2 \simeq 2.5$.

As we have seen, the contribution to the outward velocity of the ice sheet made by shear within the lowest layers of ice may or may not be negligible; but in any event, since this shearing is confined to a thin layer, its contribution to the velocity will presumably be determined by $T$. Thus we can always write $u=f(T)$, for a given point, and we shall assume the form (3), but the values of $A$ and $m$ may not be exactly those calculated from a theory of pure sliding.

One would expect from Weertman's calculation and from Glen's experiments 5 that, while $A$ would depend on roughness and temperature, $m$ would be relatively constant. $m$ is not likely to be less than about 2 and may well be considerably more. We shall use the value $2 \cdot 5$ for purposes of illustration.

( $f$ ) Reasons for the values of $T$ in Greenland. These arguments suggest an answer to the question: why is the shear stress $T$ on the bed of the Greenland ice sheet usually between about 0.3 and $I \cdot 0$ bars? On our hypotheses the shear stress is determined mainly by the roughness of the bed, the temperature and the velocity. Now, very roughly, the velocity of the ice sheet is of the same order of magnitude as the velocities of Alpine glaciers. If the roughness and the temperature were comparable in the two cases, we should therefore expect to find similar shear stresses - and the shear stresses for Alpine glaciers are in fact between about 0.5 and $\mathrm{I} \cdot 5$ bars.

This argument is similar to that given previously, but we wish to emphasize that, in the form given here, it rests on a comparison of the speeds of Alpine glaciers and of the Greenland ice sheet, rather than on a comparison of the conjectured strain-rates at the bed.

\section{Theory of Ice Sheets with a Law of Sliding}

\section{I Steady-state Solution for a Horizontal Bed}

It is illuminating to study first the following simple two-dimensional problem (Fig. 4). An ice sheet rests on a horizontal bed of uniform roughness and temperature, and the rate of accumulation $a$ is uniform over the surface. The problem is to calculate the steady-state profile. We take the origin at the centre of the bed and consider the right-hand half of the ice sheet.

For a steady state, and assuming incompressibility, we have

$$
a x=h u \text {. }
$$

We assume the law of sliding (3). $T$ is given approximately ${ }^{8}$ by

Therefore

$$
T=\rho g h \alpha=-\rho g h \frac{d h}{d x} \quad\left(\frac{d h}{d x} \ll \mathrm{I}\right) .
$$

$$
a x=h\left(-\frac{\rho g h}{A} \frac{d h}{d x}\right)^{m}
$$

or

$$
\frac{A}{\rho g} a^{\mathrm{I} / m} \int_{x}^{L} x^{\mathrm{I} / m} d x=\int_{0}^{h} h^{\mathrm{I}+\mathrm{I} / m} d h,
$$


where $L$ is the half-width. Hence we have

$$
h^{2+\mathrm{I} / m}=\frac{2 m+\mathrm{I}}{m+\mathrm{I}} \cdot \frac{A}{\rho g} \cdot a^{\mathrm{I} / m}\left(L^{\mathrm{x}+\mathrm{r} / m}-x^{\mathrm{x}+\mathrm{I} / m}\right) \quad(x>0)
$$

as the equation of the profile (the region very near $x=L$ where $|d h / d x|$ becomes large being excluded). In terms of the height $H_{\mathrm{o}}$ at the centre the profile may be written in the form

or, with $m=2 \cdot 5$,

$$
\left(\frac{h}{H_{\mathrm{o}}}\right)^{2+\mathrm{x} / m}+\left(\frac{x}{L}\right)^{\mathrm{I}+\mathrm{x} / m}=\mathrm{I} \quad(x>0)
$$

$$
\left(\frac{h}{H_{\mathrm{o}}}\right)^{2 \cdot 4}+\left(\frac{x}{L}\right)^{\mathrm{x} \cdot 4}=\mathrm{I} \quad(x>0)
$$

which is plotted in Fig. 4 .

If we allow $m$ to approach infinity, the velocity $u$ will be zero up to a critical stress $A$, and, when $T=A, u$ can take any value. Thus $m \rightarrow \infty$ corresponds to the older theory ${ }^{\mathrm{r}, 8}$ in which $T$ was taken as constant everywhere on the bed of a moving ice sheet. Putting $m \rightarrow \infty$ in equation (7) gives

$$
h^{2}=\frac{2 A}{\rho g}(L-x) \quad(x>0),
$$

which is the parabolic profile deduced on the earlier theory. It will be noticed that for finite $m$ the profile (7) depends on the accumulation, but that as $m \rightarrow \infty$ this dependence vanishes. This is why in the approximation of constant $T$ it was possible to calculate the profile without a knowledge of the accumulation. We see now that, for an ice sheet of given width, $h \propto a^{\mathrm{I} /(2 m+\mathrm{I})}$ or, with $m=2 \cdot 5, h \propto a^{\mathrm{I} / 6}$. Thus even with a realistic value of $m$ the profile is still only slightly dependent on the accumulation.

It may seem surprising at first sight that equation (I0), which was originally derived from considerations of statical equilibrium, should be deducible from equation (4), which is an equation of conservation of volume. The reason is that we have in fact ensured statical equilibrium in our model by introducing equation (5).

For $m$ infinite the complete profile consists of parts of two parabolas, one for $x<0$ and one for $x>0$; there is thus a sharp ridge at $x=0$ which necessitates a special treatment for this region. It is satisfactory to see that, with $m$ finite, the sharp ridge disappears.*

\subsection{Steady-state Solution for the General Two-dimensional Case}

We now consider the following more general two-dimensional problem (Fig. 5); an ice sheet on a bed of given profile. The roughness and temperature of the bed, and the rate of accumulation, are supposed given at each point: to calculate the steady-state profile of the upper surface.

Let the bed be given by $f(x)$ and the upper surface by $H(x)$. If the thickness is $h(x)$, we have

$$
H=h+f \text {. }
$$

We assume that $|d H| d x \mid \ll \mathrm{I}$ and $|d f| d x \mid \ll \mathrm{I}$. Let us assume that the position $(x=0)$ and the height of the highest point of the ice sheet are known in advance (we shall remove this stipulation later). Then, if the rate of accumulation is $a(x)$, one can evaluate

$$
\int_{0}^{x} a d x=I(x) \text { say. }
$$

* We have assumed in the above calculation that the velocity of sliding is not dependent on the hydrostatic pressure. If we took instead of (3) the law

$$
u=\frac{\mathrm{I}}{h}\left(\frac{T}{A}\right)^{m},
$$

the effect would be to change $\left(h / H_{0}\right)^{2+1 / m}$ in equation $(8)$ to $\left(h / H_{0}\right)^{2}$. Bodvarsson 9 has based a theory on the law of sliding $u \propto T / h$, which is the special case of the above equation with $m=\mathrm{I}$. In order to obtain solutions he assumes that the accumulation and ablation vary linearly with altitude, thus introducing a firn line. We have not adopted this theory, mainly because it seems more realistic to take a higher power of $m$ than $\mathrm{r}$. 
For a steady state equation (6) is replaced by

and hence

$$
I(x)=h\left(\frac{\rho g h \alpha}{A}\right)^{m}, \quad \alpha=-\frac{d H}{d x},
$$

$$
\delta H=-\frac{\rho g}{A} \cdot \frac{I^{\mathrm{x} / m}}{(H-f)^{\mathrm{I}+\mathrm{r} / m}} \delta x .
$$

We assume that $A, I$ and $f$ are known functions of $x$, and so the profile of the upper surface may be obtained by step-by-step integration outwards from $x=0$ in both directions.

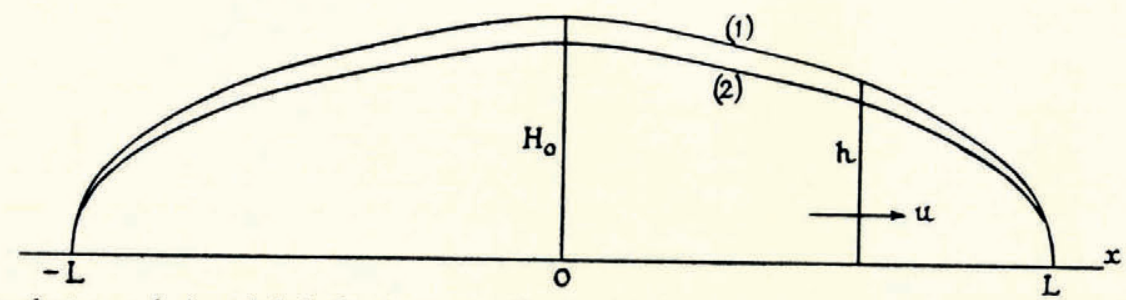
Fig. 4. Ice sheet on a horizontal bed. Steady state profiles for (1) the 2-dimensional problem, (2) a circular base. To scale
but with vertical exaggeration

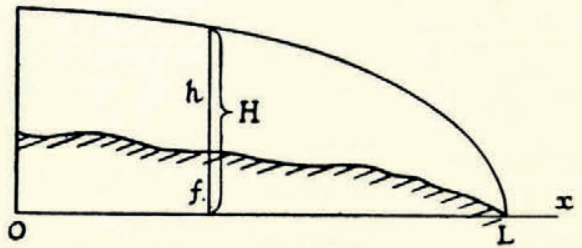

Fig. 5. Two-dimensional ice sheet on a non-horizontal bed. Not to scale

If, alternatively, the position of the edge of the ice sheet is known in advance, but not the position of the highest point, one could proceed as follows. Guess the position of the highest point. Evaluate $I(x)$. Integrate in from opposite sides of the ice sheet. The two profiles would not agree at the highest point; the position of the highest point would therefore be adjusted and the process repeated; and so on by successive approximation.

Of course the practical difficulty about the integration is to know the value of $A$ to put in at each point. In the present state of knowledge one probably has to assume $A$ is constant, faut de mieux.*

\subsection{The Effect of the Third Dimension}

The two preceding problems have been two-dimensional in that the lines of flow have been assumed to be parallel in plan view. We can obtain some insight into what happens in the three-dimensional case, where the lines of flow are not parallel in plan view, by considering an ice sheet which flows out radially from the centre. We suppose that the bed is horizontal

${ }^{*}$ Glen, ${ }^{10}$ Bull ${ }^{3}$ and Robin ${ }^{11}$ have discussed reasons for variations of $T$. Orvig ${ }^{12}$ has found that in the Barnes Ice Cap in Baffin Island $T$ appears to increase with $u$, but not invariably. ${ }^{23}$ In this connexion it is interesting to see that the values of $T$ in the north of Greenland calculated by Bull 3 are smaller towards the east coast than in the centre, even though, as Bull points out, the velocity must be greater towards the coast. This must mean that near the coast either the bed is smoother or its temperature is greater. Now the heat produced by friction at the bed will be greatest near the coast, owing to the higher velocity, and this leads to two possibilities: $(a)$ if the basal ice is already at the pressure melting point, more friction heat will produce more melt water and therefore more lubrication, as Bull mentions; $(b)$ if the basal ice is not at the pressure melting point more friction heat will produce a higher temperature. In either case, therefore, the friction heat could be invoked to explain the decrease in $T$. 
and of uniform roughness and temperature, and that the rate of accumulation $a$ is uniform over the surface. Then, proceeding as in Section $3 \cdot 1$, we find the profile to be

$$
h^{2+\mathrm{r} / m}=\frac{2 m+\mathrm{I}}{m+\mathrm{I}} \cdot \frac{A}{\rho g}\left(\frac{a}{2}\right)^{\mathrm{I} / m}\left(R^{\mathrm{I}+\mathrm{I} / m}-r^{\mathrm{I}+\mathrm{I} / m}\right),
$$

where $r$ is the distance from the centre and $R$ is the radius of the perimeter.

Let us now compare two ice sheets on horizontal beds of identical roughness and temperature: ( 1 ) is rectangular in plan view, being very long in one direction and of width $2 L$ in the other; (2) is circular in plan view and of radius $R=L$. Denoting the heights at corresponding points by $h_{\mathrm{I}}$ and $h_{2}$ respectively, we have, from equations (7) and (13),

or, with $m=2 \cdot 5$,

$$
\begin{aligned}
& h_{2}=2^{-\mathrm{r} /(2 m+\mathrm{r})} h_{1}, \\
& h_{2}=0 \cdot 89 h_{\mathrm{r}} .
\end{aligned}
$$

Thus the effect of the divergence of the flow lines in plan view is merely to reduce the height by I I per cent everywhere (Fig. 4). We note that when $m \rightarrow \infty, h_{2}=h_{\mathrm{r}}$; thus in this approximation the divergence of the flow lines has no effect at all on the profile. This agrees with the result obtained on the older theory with $T$ constant. The effect on the velocity is more pronounced. Denoting velocities at corresponding points of the two ice sheets by $u_{\mathrm{r}}$ and $u_{2}$, we find

or, with $m=2 \cdot 5$,

$$
\begin{aligned}
& u_{2}=2^{-2(m+1) /(2 m+\mathrm{r})} u_{\mathrm{I}}, \\
& u_{2}=0.45 u_{\mathrm{I}} .
\end{aligned}
$$

From these results for a circular ice sheet we may draw the conclusion that, in the general case of an ice sheet of irregular outline, the divergence of the flow lines in plan view has little effect on the profile, changing the height by not more than about io per cent. This compares with the change of o per cent obtained on the older theory. One may therefore expect to achieve a reasonably correct result by using the method of Section 3.2 and integrating along a flow line, disregarding the divergence of the flow lines.

\section{$3 \cdot 4$ Non-steady State}

(a) General. Suppose we are given the form of the upper surface of an ice sheet, which is not necessarily in a steady state, and suppose we are given its thickness $h$ and the rate of accumulation $a$ at every point. Suppose also that the law of sliding on the bed is known at each point. Then it is possible, in principle, to calculate the rate of increase or decrease of the thickness at every point as follows.

First map the contours of the upper surface and draw the lines perpendicular to them. These are the flow lines in plan view. Then, $\alpha$ and $h$ being known, $T$ can be calculated, and hence $u$, from the law of sliding. Let $q$ be the volume of ice flowing per unit time between two given flow lines a small distance $s$ apart. Then

$$
q=u h s
$$

$q(x)$ may be calculated, and hence $\partial q / \partial x$, where $x$ is measured along the streamlines. The condition for incompressibility is

$$
\frac{\mathrm{I}}{s} \frac{\partial q}{\partial x}+\frac{\partial h}{\partial t}=a
$$

Thus, since $a$ is known, $\partial h / \partial t$ may be calculated.

(b) Perturbation method. When the actual profile is only slightly different from the steadystate profile it is a simplification to work in terms of the difference between them. This development is due to Weertman* who has analysed the two-dimensional case where the bed of the ice sheet is of uniform slope and roughness. ${ }^{14}$ In the following treatment the slope of the bed and its roughness are allowed to vary from point to point; it turns out, rather interestingly, that the variation of slope has no effect at all on Weertman's result. A proper choice of variables is essential if heavy algebra is to be avoided.

* Bodvarsson has also exploited perturbation methods in his theory already mentioned. 
Since we are considering a two-dimensional problem, let $s=\mathrm{I}$. Let $\alpha$ be the slope of the upper surface. Steady-state quantities are denoted by the suffix 0 and small perturbations from the steady state by the suffix $\mathrm{I}$. Thus

$$
q=q_{\mathrm{o}}+q_{\mathrm{x}}, \quad h=h_{\mathrm{o}}+h_{\mathrm{r}}, \quad \alpha=\alpha_{\mathrm{o}}+\alpha_{\mathrm{I}} .
$$

From equation (14), remembering that $d q_{\mathrm{o}} / d x=a$, we have

Now, from equation (I I),

$$
\frac{\partial h_{\mathrm{r}}}{\partial t}=\frac{\partial h}{\partial t}=a-\frac{\partial q}{\partial x}=-\frac{\partial q_{\mathrm{r}}}{\partial x}
$$

and hence

$$
q=\left(\frac{\rho g}{A}\right)^{m} h^{m+\mathrm{r}} \alpha^{m}
$$

$$
q_{\mathrm{I}}=q_{\mathrm{o}}\left\{(m+\mathrm{I}) \frac{h_{\mathrm{I}}}{h_{\mathrm{o}}}+m \frac{\alpha_{\mathrm{r}}}{\alpha_{\mathrm{o}}}\right\} .
$$

Therefore, differentiating this equation with respect to $x$, and putting $d q_{\mathrm{o}} / d x=a$, we obtain from equation (15) the following equation for the behaviour of the perturbation $h_{1}$ :

$$
\frac{\partial h_{\mathrm{r}}}{\partial t}=-B h_{\mathrm{r}}-C \frac{\partial h_{\mathrm{r}}}{\partial x}+D \frac{\partial^{2} h_{\mathrm{r}}}{\partial x^{2}}
$$

where $B, C, D$ are quantities fixed by the steady-state parameters as follows,

and where $\phi_{0}=-d h_{\mathrm{o}} / d x$.

$$
\begin{aligned}
B & =(m+\mathrm{I})\left(u_{\mathrm{o}} \phi_{\mathrm{o}}+a\right) / h_{\mathrm{o}} \\
C & =(m+\mathrm{I}) u_{\mathrm{o}}-\frac{m a}{\alpha_{\mathrm{o}}}+\frac{m h_{\mathrm{o}} u_{\mathrm{o}}}{\alpha_{\mathrm{o}}{ }^{2}} \frac{d \alpha_{\mathrm{o}}}{d x} \\
D & =\frac{m h_{\mathrm{o}} u_{\mathrm{o}}}{\alpha_{\mathrm{o}}}
\end{aligned}
$$

It will be noticed that, in this approach, $d A / d x$, which measures the variation in bed roughness, does not appear in the expressions (I8) and neither does the slope of the bed or its derivatives. However, if we wish to write $d \alpha_{0} / d x$ in terms of the other steady-state parameters we may do so (by writing down the equation for $q_{\mathrm{o}}$ and differentiating with respect to $x$ ), but at the expense of bringing in $d A / d x$. If we do this the expression for $C$ becomes

and so, for constant bed roughness,

$$
C=\frac{\mathrm{I}}{\alpha_{\mathrm{o}}}\left\{(m+\mathrm{I})\left(\alpha_{\mathrm{o}}+\phi_{\mathrm{o}}\right) u_{\mathrm{o}}-(m-\mathrm{I}) a+\frac{m h_{\mathrm{o}} u_{\mathrm{o}}}{A} \frac{d A}{d x}\right\},
$$

$$
C=\frac{\mathrm{I}}{\alpha_{0}}\left\{(m+\mathrm{I})\left(\alpha_{0}+\phi_{0}\right) u_{0}-(m-\mathrm{I}) a\right\},
$$

which is equivalent to Weertman's result.

As Weertman has pointed out, the three terms on the right-hand side of equation ( 17 ) have simple interpretations. The first represents an exponential decay of an initial disturbance with time constant $B^{-1}$; the second represents a wave travelling with velocity $C$; the third represents an outwards spreading of a disturbance in accordance with the diffusion equation. A simple argument leading to the above expression $m h_{\mathrm{o}} u_{\mathrm{o}} / \alpha_{\mathrm{o}}$ for the diffusion coefficient $D$ is given in the Appendix (p. 507).

If, following Weertman, we change from $(x, t)$ to a coordinate system $\left(x^{\prime}, t^{\prime}\right)$ moving to the right with velocity $C$,

equation ( I 7 ) simplifies to

$$
x^{\prime}=x-C t, \quad t^{\prime}=t,
$$

$$
\frac{\partial h_{\mathrm{I}}}{\partial t^{\prime}}=-B h_{\mathrm{I}}+D \frac{\partial^{2} h_{\mathrm{r}}}{\partial x^{\prime 2}} .
$$

A solution of (2I) which is of interest in connexion with the Antarctic ice sheet is

$$
h_{\mathrm{I}}=k_{\mathrm{I}} \exp \left(-B t^{\prime}\right)\left\{\mathrm{I}+k_{2} \exp \left(-4 \pi^{2} D / \lambda^{2}\right) t^{\prime} \sin \left(2 \pi x^{\prime} / \lambda\right)\right\},
$$


where $k_{\mathrm{I}}, k_{2}$ and $\lambda$ are constants. At $t^{\prime}=0$ the surface is displaced from the steady state by a uniform amount, and, in addition, there are sinusoidal waves on it, thus

$$
h_{\mathrm{I}}=k_{\mathrm{I}}\left\{\mathrm{I}+k_{2} \sin \left(2 \pi x^{\prime} / \lambda\right)\right\} \quad\left(t^{\prime}=\mathrm{o}\right) \text {. }
$$

The uniform perturbation $k_{\mathrm{r}}$ dies away with a time constant $t_{\mathrm{r}}=B^{-\mathrm{I}}$, while the amplitude of the sine waves dies away with a time constant

$$
t_{2}=\left(B+\frac{4 \pi^{2} D}{\lambda^{2}}\right)^{-x} .
$$

It is evident that for a general initial displacement of the surface each Fourier component of the displacement would behave in the way just calculated.

Applying this to central Antarctica we put $m=2 \cdot 5, h_{0}=3,000 \mathrm{~m} ., u_{0}=20 \mathrm{~m} . / \mathrm{yr} ., \alpha_{0}=\phi_{0}=$ $3 \times 10^{-3}, a=0 \cdot 10 \mathrm{~m} . / \mathrm{yr}$. and find $t_{\mathrm{r}}=5,000 \mathrm{yr}$. Values of $t_{2}$ for various wavelengths are as follows :

$$
\begin{array}{rccrrr}
\lambda: & 3 & \text { I } 4 & 40 & \text { I } 40 & 8,000 \mathrm{~km} . \\
t_{2}: & 0 \cdot 005 & 0 \cdot 1 & \text { I } & \text { I0 } & 5,000 \mathrm{yr} .
\end{array}
$$

The approximations of the theory break down when $\lambda<h$; thus we conclude that waves (Fourier components) with $\lambda$ between about 3 (or less) and $40 \mathrm{~km}$. will have lifetimes of less than one year, while a large scale perturbation of the whole ice sheet will last for $\sim 5,000 \mathrm{yr}$.which is long compared with the time over which the rate of accumulation can be considered as constant. Surface waves in the range $\lambda=5$ to $30 \mathrm{~km}$. have been observed during the crossing of the Trans-Antarctic Expedition. Since they cannot have been built up by drift in as little as one year, they cannot, according to the present theory, be departures from the equilibrium profile, and are presumably caused by mountains buried beneath the ice. ${ }^{15}$

It is interesting that the kinematic wave velocity $C$ can be negative if $a$ is sufficiently large or $u_{\mathrm{o}}$ sufficiently small. Putting in rough values for Antarctica one finds that $C$ is negative up to distances of about $200 \mathrm{~km}$. from the centre, so that up to this radius waves are propagated back towards the centre.*

\subsection{The Effect of Bed Topography on the Upper Surface}

The form of the upper surface of an ice sheet must to some extent reflect the form of the bed. If $m$ were infinite, the shear stress $T$ on the bed would be equal to $A$, and if $A$ were constant $T$ would be the same everywhere. This, as we have seen, was the approximation made in the older theory. ${ }^{8}$ Since $T=\rho g h \alpha$, when $T$ is constant $h$ is proportional to $\mathrm{I} / \alpha$. By using this extremely direct and simple relation Bourgoin ${ }^{16}$ has succeeded in making a detailed correlation between the very slight relief of the surface of the Greenland ice sheet and the relief of the bed measured by seismic sounding. He found that an unevenness of the surface of as little as ro $\mathrm{m}$. between two points $30 \mathrm{~km}$. apart denoted the presence of a hill in the bedrock about $35^{\circ} \mathrm{m}$. high. The essence of Bourgoin's method is the simple fact that when the slope is greatest the thickness is least, and vice versa-and hence the points of inflexion of the surface correspond approximately to maxima and minima of the altitude of the bedrock. A further demonstration of the essential correctness of this idea is provided by Robin's observations in Dronning Maud Land, Antarctica. ${ }^{\text {II }}$ Robin discovered by seismic sounding that steps on the upper surface of the ice sheet corresponded to ridges in the bed, the highest point of the submerged ridge lying under the zone of maximum surface slope-and this, as he points out, is what one would expect from the simple theory.

This theory, which assumes that $m$ is infinite and therefore $\mathcal{T}$ constant, can now be improved by using the more general relation (3), in which $m$ is finite and which allows for changes in $T$. Of course an observed irregularity on the surface might conceivably be due, not to a hill or valley on the bed, but to a local variation of bed roughness or temperature.

* Weertman's conclusion that the wave velocity is about $(3 m+r)$ times the surface velocity seems invalid, for an interesting reason. To obtain it he approximates the upper surface by a parabola (which is a good approximation); but, as we have seen in equation (10), this is equivalent to putting $m$ infinite; the method is thus not self-consistent. 
However, Bourgoin and Robin's results encourage one to think that the topography of the bed is the chief factor which determines the form of the surface in any local region. Let us therefore now calculate the perturbation of the upper surface caused by the presence of a small ridge on the bed.

Let the ice sheet be two-dimensional, let it rest on a bed whose slope varies with $x$, and let there be somewhere on the bed a ridge of limited width and of height $p(x)$, which lies perpendicular to the direction of flow (Fig. 6). For a valley $p(x)$ is negative. We compare this,

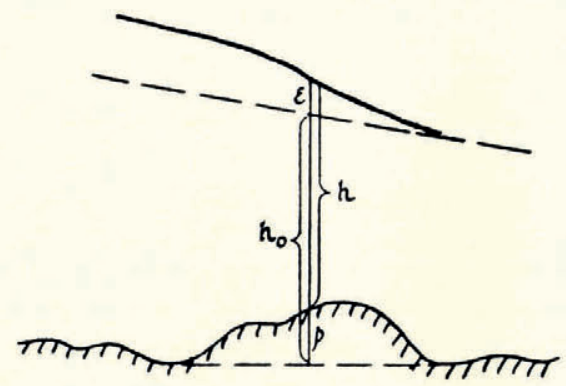

Fig. 6. Perturbation $\epsilon(x)$ of the upper surface of an ice sheet caused by a small ridge $p(x)$ on the bed. It is shown that $\epsilon(x)$ is in fact small compared with $p(x)$ and that the perturbation of the surface profile is approximately proportional to the integral
of the bed profile

the real ice sheet, with a hypothetical ice sheet, called the "reference sheet", resting on a bed which is identical with the real bed in all respects except that it does not possess the ridgewhere the ridge would have been the bed is horizontal. The thickness of the real ice sheet is $h(x)$, and the slope of its upper surface is $\alpha(x)$. The corresponding quantities for the reference sheet are $h_{0}(x), \alpha_{0}(x)$; in general, quantities referring to the reference sheet have suffix $o$. It turns out that it is not possible to make the reference sheet identical with the real sheet both far upstream and far downstream of the ridge. We therefore stipulate that $h_{0}(x)=h(x)$ sufficiently far downstream of the ridge, thereby ensuring that the edges of the ice sheets coincide. Let $p \ll h_{\mathrm{o}}$, and write

$$
h=h_{\mathrm{o}}+h_{\mathrm{I}} \quad\left(h_{\mathrm{I}} \ll h_{\mathrm{o}}\right), \quad \alpha=\alpha_{\mathrm{o}}+\alpha_{\mathrm{I}} \quad\left(\alpha_{\mathrm{I}} \ll \alpha_{\mathrm{o}}\right) .
$$

The ice sheet is not restricted to be in a steady state. Integrating equation (14) from the centre of the ice sheet $(x=0)$, with $s=\mathrm{I}$, we have

$$
q=\int_{0}^{x} a d x-\frac{\partial}{\partial t} \int_{0}^{x} h d x .
$$

The first term on the right-hand side is the net addition of material between $O$ and $x$, and the second is the rate of decrease of volume of the ice between $O$ and $x$. The presence of the ridge on the bed has no effect on the first term, except in so far as it changes the position of the centre of the ice sheet, and we shall ignore this very small effect. Likewise we may assume that the presence of a single ridge has no significant effect on the second term, for it is hardly likely that it can seriously affect the wastage of the whole ice sheet between $O$ and $x$ (this is certainly true when both the real sheet and the reference sheet are in steady states). Therefore we write

Hence, from equation ( 16 )

$$
q=q_{\mathrm{o}}
$$

$$
h^{m+1} \alpha^{m}=h_{\mathrm{o}}{ }^{m+1} \alpha_{\mathrm{o}}{ }^{m},
$$


and thus

Now

$$
(m+\mathrm{I}) \frac{h_{\mathrm{r}}}{h_{\mathrm{o}}}+m \frac{\alpha_{\mathrm{I}}}{\alpha_{\mathrm{o}}}=0 .
$$

and so

$$
\begin{gathered}
\alpha_{\mathrm{o}}=-\frac{d h_{\mathrm{o}}}{d x} \text { and } \alpha_{\mathbf{I}}=-\frac{d}{d x}\left(h_{\mathrm{I}}+p\right), \\
\frac{d h_{\mathrm{I}}}{d x}+\frac{m+\mathrm{I}}{m} \frac{h_{\mathrm{I}}}{h_{\mathrm{o}}} \frac{d h_{\mathrm{o}}}{d x}+\frac{d p}{d x}=0 .
\end{gathered}
$$

This integrates to

$$
h_{\mathrm{o}}{ }^{(m+\mathrm{x}) / m} h_{\mathrm{I}}=-\int_{0}^{p} h_{\mathrm{o}}^{(m+\mathrm{r}) / m} d p,
$$

since $h_{\mathrm{r}}=\mathrm{o}$ sufficiently far downstream of the ridge. Since $h_{\mathrm{o}}$ changes only slightly over the region of the ridge, which is of limited width, we may take it outside the integration. Thus we obtain

$$
h_{\mathrm{I}}(x) \simeq-p(x) .
$$

The change in height of the surface over the ridge is $h_{\mathrm{r}}+p=\epsilon$, say; our result therefore means that $\epsilon$ is an order of magnitude less than $p$. In other words the disturbance on the surface is much smaller than the ridge on the bed.

The change in slope caused by the ridge is found from equation (23); thus

$$
\frac{\alpha_{\mathrm{I}}}{\alpha_{\mathrm{o}}} \simeq \frac{m+\mathrm{I}}{m} \cdot \frac{p}{h_{\mathrm{o}}} \simeq \mathrm{I}_{\mathrm{I}} \cdot 4 \frac{p}{h_{\mathrm{o}}} \quad(\text { for } m=2 \cdot 5) .
$$

$\alpha_{\mathrm{I}} / \alpha_{\mathrm{o}}$ is therefore of first order in $p / h_{0}$, in contrast to $\epsilon / h_{\mathrm{o}}$ which, as we have seen, is an order of magnitude smaller than $p / h_{\mathrm{o}}$.

The change $T_{1}$ in the shear stress $T_{0}$ on the bed is found by returning to (22) and writing

$$
h T^{m}=h_{\mathrm{o}} T_{\mathrm{o}}{ }^{m} \text {, }
$$

so that

$$
\frac{T_{\mathrm{I}}}{T_{\mathrm{o}}}=-\frac{\mathrm{I}}{m} \cdot \frac{h_{\mathrm{I}}}{h_{\mathrm{o}}} \simeq \frac{\mathrm{I}}{m} \cdot \frac{p}{h_{\mathrm{o}}} \simeq 0 \cdot 4 \frac{p}{h_{\mathrm{o}}} .
$$

Thus a mountain ridge $300 \mathrm{~m}$. high covered by ice $3,000 \mathrm{~m}$. thick shows its presence by causing a change in height of the surface small compared with $300 \mathrm{~m}$. (as verified by Bourgoin's results). On the other hand it causes a change in surface slope of i 4 per cent and a change in $T$ of 4 per cent. By putting $m$ infinite we see that on the older theory the corresponding figures would be ro per cent and o per cent.

A change of $T$ of the same sign as predicted by equation (27) is in fact shown by the observations in Bourgoin's paper ${ }^{16}$ (although he regards the variation in $T$ as within the experimental error). Referring to his Figure 5 and Table III one notices that the high values of $T$ (proportional to $h_{0}$ in his notation) are all associated with high places on the bed. The amount of the observed variation in $T$ (about 15 per cent), which of course includes the observational error, is a good deal larger than the theoretical variation (about 4 per cent).

Since $\alpha_{0}$ and $h_{0}$ are approximately constant over the region of the ridge, equation (25) means that, in any local region, the profile of the bed $p(x)$ is obtained by differentiating the profile of the surface perturbation; and likewise, one obtains the surface perturbation by integrating the bed profile; thus

$$
\epsilon \simeq \frac{m+\mathbf{I}}{m} \cdot \frac{\alpha_{0}}{h_{0}} \int_{x}^{x_{0}} p d x,
$$

since $\epsilon=0$ downstream, $x_{0}$ being a point downstream from the ridge. Of course, over large distances the relation is not as simple as this because $\alpha_{0}$ and $h_{0}$ can no longer be considered 
constant. This simple result for a local region is valid both for $m$ finite and $m$ infinite, the only difference introduced by the new theory being in the constant of proportionality, $(m+\mathbf{I}) / m$ instead of $\mathrm{I}$.

In order to deduce the profile $p(x)$ of a buried mountain ridge or valley from the change $\alpha_{\mathrm{I}}(x)$ that it produces in the slope of the surface, we write (25) as

$$
p(x) \simeq \frac{m}{m+\mathrm{I}} \cdot \frac{h \alpha_{\mathrm{I}}}{\alpha_{\mathrm{o}}} \simeq 0 \cdot 7 \frac{h \alpha_{\mathrm{I}}}{\alpha_{0}} .
$$

The value of $p$ deduced by putting $m$ infinite is $h \alpha_{\mathrm{I}} / \alpha_{0}$. Thus with the new theory we deduce values of $p$ which are 70 per cent of those deduced on the older theory. Perhaps the simplest way of explaining this result is as follows. We have

$$
\frac{h_{\mathrm{I}}}{h_{\mathrm{o}}}=-\frac{\alpha_{\mathrm{I}}}{\alpha_{\mathrm{o}}}+\frac{T_{\mathrm{I}}}{T_{\mathrm{o}}}
$$

Suppose $\alpha$ is observed to increase locally by ro per cent. This produces an increase of 3 per cent in $T$, instead of o per cent as previously guessed; and so, instead of indicating a ro per cent decrease in thickness, it now indicates only a 7 per cent decrease.

\section{Application to Glaciers and Ice Sheets in which Differential Motion is Important}

The theory of Ref. 2, which is based on equation (2), gives essentially the local differential motions and stresses within a parallel-sided glacier (or ice sheet), the influence of the valley sides being neglected. It contains as a parameter the longitudinal strain-rate $\dot{\epsilon}_{x}$ of the glacier (in Ref. $2\left|\dot{\epsilon}_{x}\right|$ is denoted by $r$ ). $\dot{\epsilon}_{x}$ in the theory is independent of depth and is an independent variable to be determined empirically for each case. On the other hand, in the theory of the perfectly plastic glacier ( $n$ infinite) $\dot{\epsilon}_{x}$ is given explicitly by the equation

$$
\dot{\epsilon}_{x}=\frac{a}{h}+\bar{u}_{\kappa} \cot \alpha,
$$

where $a$ is the rate of accumulation, $h$ is the thickness, $\bar{u}$ is the forward velocity of the ice averaged through the thickness, $\kappa$ is the curvature of the surface, and $\alpha$ is the slope of the surface. By introducing the sliding law (3) it is now possible to derive an explicit expression for $\dot{\epsilon}_{x}$, even with $n$ finite, and thus to complete the theory.

In many glaciers both internal differential motion and sliding on the bed may be important, and our theory must include both. Now, as we have said, in the theory with $n$ finite $\dot{\epsilon}_{x}$ is independent of depth. To calculate its value we may therefore work with any depth we please, and it is most convenient to evaluate it at the bed of the glacier where $\dot{\epsilon}_{x}$ is simply $\partial u / \partial x, u$ being the velocity of sliding. This is the important step in the argument. Of course, in a real glacier $\dot{\boldsymbol{\epsilon}}_{\boldsymbol{x}}$ will doubtless vary through the thickness (for an example, see Ref. I 7), but since this variation is neglected in the theory of differential motion that we are using we may as well write $\dot{\boldsymbol{\epsilon}}_{\boldsymbol{x}}=\partial u / \partial x$.

We have

$$
u=\left(\frac{T}{A}\right)^{m}, \quad T=\rho g h \sin \alpha .
$$

If we neglect any variation in $A$ and $m$, differentiation gives

$$
\dot{\epsilon}_{x}=\frac{\partial u}{\partial x}=\frac{m u}{h} \frac{\partial h}{\partial x}+m u \kappa \cot \alpha,
$$

where $\kappa=\partial \alpha / \partial x$. Now, from straightforward geometrical considerations at the upper surface we have

$$
\frac{\partial h}{\partial t}=a+v_{s}-u_{s} \frac{\partial h}{\partial x}
$$

where $u_{s}$ and $v_{s}$ are the velocity components at the surface, respectively parallel to the bed 
and perpendicularly upwards from it. We also know that $v_{s}=-h \dot{\epsilon}_{x}$. Therefore, using this equation for $v_{s}$ and eliminating $\partial h / \partial x$ between (30) and (3I) we find

$$
\dot{\epsilon}_{x}=\frac{m u}{m u+u_{s}}\left(\frac{a}{h}+u_{s} \kappa \cot \alpha-\frac{\mathrm{I}}{h} \frac{\partial h}{\partial t}\right) \text {. }
$$

For $m \rightarrow \infty$, and a steady state, this reduces to formula (29), except that $u_{s}$ appears in place of $\bar{u}$; this is in fact a trivial difference, since in the perfect plasticity approximation the total differential velocity is small compared with the absolute forward velocity, and thus, for this case, $\bar{u} \sim u_{s}$.

The effect of a change in the width of the valley may be allowed for in an ad hoc way by writing in place of $v_{s}=-h \dot{\epsilon}_{x}$ the equation $v_{s}=-h\left(\dot{\epsilon}_{x}+\dot{\epsilon}_{z}\right)$, where $\dot{\epsilon}_{z}$ is the transverse strain-rate, so that volume is conserved. If the width of the valley is $w$, we may write $\dot{\epsilon}_{z}=(\bar{u} / w)(d w / d x)$, and this leads to the following modification of equation (32):

$$
\dot{\boldsymbol{\epsilon}}_{x}=\frac{m u}{m u+u_{s}}\left(\frac{a}{h}+u_{s} \kappa \cot \alpha-\frac{\mathrm{I}}{h} \frac{\partial h}{\partial t}-\frac{\bar{u}}{w} \frac{d w}{d x}\right) .
$$

Summarizing this result we may say that equation (33) is an improvement on the former equation (29) in the following respects: by allowing $m$ (and $n$ ) to be finite it takes account of the variation of shear stress on the bed; it is not restricted to a steady state; and it takes account of variations in the width of the valley. If the motion is entirely by bed slip, and if $m=2 \cdot 5$, the factor $m u /\left(m u+u_{s}\right)$ is equal to $0 \cdot 7$. If, on the other hand, bed slip and internal differential motion make equal contributions to the surface velocity, the factor is $0 \cdot 6$. Thus, provided differential motion does not provide more than half the surface velocity, one deduces values of $\dot{\epsilon}_{x}$ on the new theory which are roughly 60 or 70 per cent of the values deduced from perfect plasticity theory.

MS. received I2 May 1959

\section{REFERENCES}

I. Nye, J. F. The flow of glaciers and ice-sheets as a problem in plasticity. Proceedings of the Royal Society, Series A, Vol. 207, No. 1091 , 1951, p. 554-72.

2. Nye, J. F. The distribution of stress and velocity in glaciers and ice-sheets. Proceedings of the Royal Society, Series A, Vol. 239, No. 12 16, 1957, p. 11 3-33.

3. Bull, C. Observations in north Greenland relating to theories of the properties of ice. Fournal of Glaciology, Vol. 3, No. 21, 1957, p. 67-72.

4. Robin, G. de Q. Ice movement and temperature distribution in glaciers and ice sheets. Fournal of Glaciology, Vol. 2, No. 18, 1955, p. 523-32.

5. Glen, J. W. The creep of polycrystalline ice. Proceedings of the Royal Society, Series A, Vol. 228, No. 1175, I 955 , p. $519-38$.

6. Rigsby, G. P. Effect of hydrostatic pressure on velocity of shear deformation of single ice crystals. Fournal of Glaciology, Vol. 3, No. 24, 1958, p. 273-78.

7. Weertman, J. On the sliding of glaciers. Fournal of Glaciology, Vol. 3, No. 21, 1957, p. 33-38.

8. Nye, J. F. A method of calculating the thicknesses of the ice-sheets. Nature, Vol. 169, No. 4300, 1952, p. 529-30.

9. Bodvarsson, G. On the flow of ice-sheets and glaciers. Fökull, Âr. 5, 1955, p. I-8.

10. Glen, J. W. Depth measurements of ice caps and the theory of glacier flow. [Comments on paper by Holtzscherer and Robin.] Geographical fournal, Vol. 120, Part 2, 1954, p. $201-02$.

I 1. Robin, G. de Q. Glaciology. III. Seismic shooting and related investigations. Norwegian-British-Swedish Antarctic Expedition, 1949-52. Scientific Results. (Oslo, Norsk Polarinstitutt), Vol. 5, 1958, p. 126-27.

12. Orvig, S. On the variation of the shear stress on the bed of an ice cap. Journal of Glaciology, Vol. 2, No. 14, 1953, p. 242-47.

13. Ward, W. H. Comments on Dr. Orvig's paper. Journal of Glaciology, Vol. 2, No. 14, 1953, p. $247-48$.

14. Weertman, J. Traveling waves on glaciers. Union Géodésique et Géophysique Internationale, Association Internationale d'Hydrologie Scientifique, Symposium de Chamonix, I6-24 sept. 1958, Physique du mouvement de la glace, 1958, p. 162-68.

15. Nye, J. F. Surface topography of the Antarctic ice sheet. Nature, Vol. 184, No. 4689, 1959, p. $786-87$.

16. Bourgoin, J.-P. Quelques caractères analytiques de la surface et du socle de l'inlandsis groenlandais. Annales de Géophysique, Tom. 12, No. i, 1956, p. 75-83.

17. Nye, J. F. The deformation of a glacier below an ice fall. Fournal of Glaciology, Vol. 3, No. 25, 1959, p. 387-408. 


\section{APPENDIX}

Simple Argument giving the Value of the Diffusion Coefficient for a Surface Disturbance The value of $D$ in equation ( 17 ) is found in the text to be $m h_{0} u_{0} / \alpha_{0}$. A simple, but not rigorous, argument leading
to the same result is as follows.

Suppose there is a surface disturbance as shown in Fig. 7. The mean slope is $\alpha_{0}$, the slope at $P$ is $\alpha_{0}-\alpha_{1}$ and at

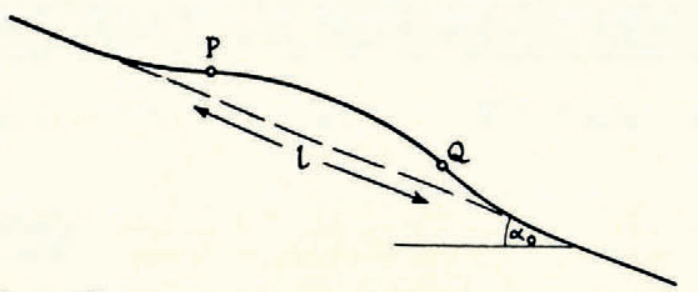

Fig. 7. Illustrating the outwards diffusion of a surface disturbance

$Q$ is $\alpha_{0}+\alpha_{1}$; similarly the mean velocity is $u_{0}$, the velocity at $P$ is $u_{0}-u_{1}$ and at $Q$ is $u_{0}+u_{1}$. From the law of sliding, $u \propto \alpha^{m}$ ( $h$ being approximately constant); hence

$$
\frac{u_{\mathrm{I}}}{u_{\mathrm{o}}}=m \frac{\alpha_{\mathrm{I}}}{\alpha_{\mathrm{o}}} .
$$

There is thus a horizontal strain-rate $2 u_{\mathrm{I}} / l \quad(P Q=l)$, and, associated with this, a vertical velocity of

$$
-\frac{2 u_{\mathrm{I}} h_{\mathrm{O}}}{l}=-\frac{m h_{\mathrm{o}} u_{\mathrm{O}}}{\alpha_{\mathrm{o}}} \cdot \frac{2 \alpha_{\mathrm{I}}}{l} \text {. }
$$

But $2 \alpha_{1} / l$ is the curvature of the surface between $P$ and $Q$. Therefore

$$
\frac{\partial h_{\mathrm{I}}}{\partial \ell}=\frac{m h_{\mathrm{o}} u_{\mathrm{o}}}{\alpha_{\mathrm{o}}} \frac{\partial^{2} h_{\mathrm{I}}}{\partial x^{2}} .
$$

